\title{
Risk Analysis of On-Orbit Spacecraft Refueling Concepts
}

\author{
William M. Cirillo ${ }^{1}$ \\ NASA Langley Research Center, Hampton, VA, 23666 \\ Chel Stromgren ${ }^{2}$ and Grant R. Cates ${ }^{3}$ \\ Science Application International Corporation, McLean, VA 22102
}

\begin{abstract}
On-orbit refueling of spacecraft has been proposed as an alternative to the exclusive use of Heavy-lift Launch Vehicles to enable human exploration beyond Low Earth Orbit (LEO). In these scenarios, beyond LEO spacecraft are launched dry (without propellant) or partially dry into orbit, using smaller or fewer element launch vehicles. Propellant is then launched into LEO on separate launch vehicles and transferred to the spacecraft. Refueling concepts are potentially attractive because they reduce the maximum individual payload that must be placed in Earth orbit. However, these types of approaches add significant complexity to mission operations and introduce more uncertainty and opportunities for failure to the mission. In order to evaluate these complex scenarios, the authors developed a Monte Carlo based discrete-event model that simulates the operational risks involved with such strategies, including launch processing delays, transportation system failures, and onorbit element lifetimes. This paper describes the methodology used to simulate the mission risks for refueling concepts, the strategies that were evaluated, and the results of the investigation. The results of the investigation show that scenarios that employ refueling concepts will likely have to include long launch and assembly timelines, as well as the use of spare tanker launch vehicles, in order to achieve high levels of mission success through Trans Lunar Injection.
\end{abstract}

$\begin{array}{ll} & \text { Acronyms } \\ \text { ARD } & =\text { Autonomous Rendezvous and Dock } \\ \text { DES } & =\text { Discrete Event Simulation } \\ \text { EDS } & =\text { Earth Departure Stage } \\ \text { EELV } & =\text { Evolved Expendable Launch Vehicle } \\ \text { HLV } & =\text { Heavy-lift Launch Vehicle } \\ \text { LEO } & =\text { Low Earth Orbit } \\ \text { MAST } & =\text { Manifest Assessment Simulation Technique } \\ \text { MMOD } & =\text { micrometeorite and orbital debris } \\ \text { MMT } & =\text { Mission Management Team } \\ \text { ORM } & =\text { Orbital Refueling Model } \\ \text { TLI } & =\text { Trans Lunar Injection }\end{array}$

\section{Introduction}

A S part of NASA's support of the 2009 Review of U.S. Human Space Flight Plans Committee ${ }^{1}$ options, a Idetailed analysis was undertaken to examine the viability of refueling spacecraft in Low Earth Orbit (LEO) using some form of on-orbit propellant refueling strategy. This approach is attractive because it allows a greater fraction of the mass delivered to LEO to be dedicated to the spacecraft and its associated payload, rather than to propellant. This would allow for increased overall mission performance using a given launch vehicle and/or potentially could eliminate the need to develop a new heavy-lift launch capability to support exploration.

\footnotetext{
${ }^{1}$ Senior Researcher, Space Missions Analysis Branch, MS 462, AIAA Member.

${ }^{2}$ Chief Scientist, Center for Strategic Analytics, 1710 SAIC Drive, AIAA Member.

${ }^{3}$ Program Management Consultant, Center for Strategic Analytics, 1710 SAIC Drive, Senior AIAA Member.
} 
Rather than having to launch transportation elements fully fueled, on-orbit refueling allows those elements to be launched dry, at only a fraction of the total fueled mass. Propellant is then launched on one or more additional launch vehicles and transferred to the element in Low Earth Orbit (LEO) ${ }^{2}$.

While the refueling concept is attractive from a performance perspective, there are questions concerning the integrated reliability of such a system from an overall operational perspective. To address these concerns, the authors completed an effort to look at the mission-level risks involved with such strategies, including risks associated with launch delays, transportation system failures, and on-orbit system lifetimes. A Monte Carlo based discrete-event simulation model was developed to evaluate each failure type for various refueling strategies and to develop overall probabilities of mission failure for each. Finally, these failure probabilities were compared to those for the proposed NASA Constellation architecture ${ }^{3}$.

This paper describes in Section 2 the concept of operations for on-orbit refueling. Section 3 describes the complexities and risks in these strategies. The methodology used to evaluate the mission risks for refueling systems are discussed in Section 4. Section 5 addresses the strategies that were evaluated. The results of the investigation are discussed in Section 6 and some conclusions and forward work are presented in Section 7.

\section{Concept of Operation for On-Orbit Refueling}

The simulation model that is described in this paper was designed to evaluate a broad range of possible refueling and depot strategies, as well as a range of possible exploration destinations. For the purposes of demonstrating the operation of the simulation model and the resulting analysis, one specific concept of operations is detailed in this paper. This particular concept is designed to support human lunar landing missions and involves the launch of a nonfueled (empty) Earth Departure Stage (EDS) into LEO. The basic concept of operations is shown in Figure 1.

In this concept a dry EDS is first launched into LEO. Some number of propellant carrying and refueling vehicles $[\mathrm{X}]$ are then launched serially into orbit where autonomous rendezvous and docking (ARD) with the EDS is performed. Propellant is transferred into the EDS from each refueling vehicle. When the EDS has been fully fueled, a Lunar Lander and a Crewed vehicle are then launched separately and rendezvous and dock with the EDS. The

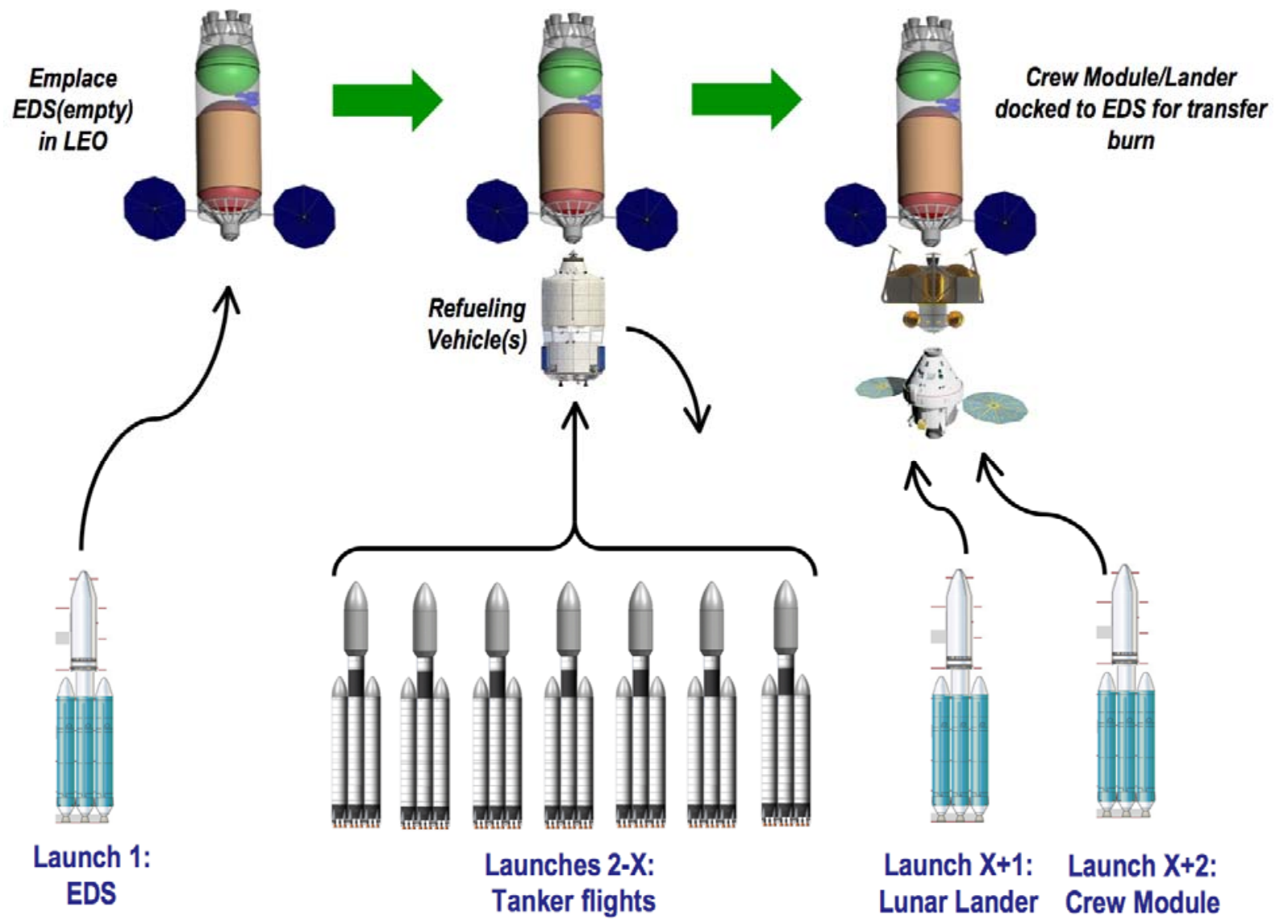

Figure 1. EDS Refueling Concept of Operations 
entire stack, including the EDS, Lander, and Crew Module are propelled to the Moon by the EDS. In this concept, the EDS acts as the on-orbit fuel depot ${ }^{4,5}$. This is attractive because no permanent on-orbit depot is required to collect and distribute fuel.

\section{Complexities and Risks in On-Orbit Refueling Strategies}

The general drawback of on-orbit refueling scenarios is that they require a greater number of launches to place the elements and propellant in orbit, as compared to launching fully fueled vehicles. The additional launches add to the complexity of operations and potentially increase the risk to the overall mission.

In the on-orbit refueling strategy described above, it is anticipated that, using an Evolved Expendable Launch Vehicle (EELV) with the capability to deliver 25 metric tons of payload to LEO, it would take between 5 and 10 planned launches to supply the required amount of propellant to orbit. The actual number of required tanker flights will depend on the desired capacity to the lunar surface and the propellant boil-off rate that can be achieved on-orbit. Such a large number of launches will add a significant amount of complexity and uncertainty to mission operations, and increases the opportunities for overall mission failure.

There are two primary areas of risk that are of concern in this scenario. The first is the large number of opportunities for mission failure. In the scenario described in Figure 1, if 8 refueling flights are required, then a total of 11 Earth to LEO launches are required, along with ten rendezvous and docking events, and eight propellant transfer events. Each of these events has the potential to disrupt the overall mission. Although each individual event may be accomplished with high reliability, the total number of events could still result in a relatively low overall mission reliability.

The second major area of risk involves the orbital lifetime limit of the system. The period over which the EDS can remain in LEO will be limited by several factors; the design lifetime of the element, the rate at which propellant will boil-off while in orbit, and the risk associated with micrometeorite and orbital debris (MMOD). Because of these factors there will be some restricted period of time in which all of the planned launches must take place. The lifetime of the EDS for a lunar mission can theoretically be extended a significant amount; however, this would involve added complexity, mass, and cost.

One option for reducing the overall mission risk is to include into the concept of operations the possibility of attempting to re-fly any tanker flights that fail during the launch and refueling process. Because of the number of tanker flights, a major portion of the overall mission risk is involved with failure to launch, rendezvous, or transfer propellant on one of these flights. Since these flights are rather generic in nature, it is possible to simply have extra launchers and spacecraft available and ready to fly. In the event that one or more of these flights is a failure, a backup can be launched to complete fueling, avoiding the failure of the overall mission. It is assumed that there is no option to re-fly any of the other three missions; the EDS, the Lunar Lander, or the Crew Module in the event of an unwanted incident. These flights involve unique elements and the loss of any one of them results in failure of the overall mission. While the ability to re-fly tanker flights may lead to fewer overall technical failures, this option actually increases the chance of EDS on-orbit lifetime failure. Any tanker re-flights that occur will stretch out the overall launch schedule, increasing the probability that the EDS on-orbit lifetime will be exceeded. In addition, the cost of having extra tanker flights constantly available for use could be considerable.

\section{Simulation Models}

The complexities that are described for on-orbit refueling scenarios make it difficult to analyze the overall mission level of reliability. There are many elements that need to work together to be successful in this scenario, and failures in any of the elements have feedbacks that cause delays or failures in other elements.

The simulation models that were used to evaluate refueling scenarios involve two components:

- Orbital Refueling Model (ORM)

- Manifest Assessment Simulation Technique (MAST)

These two components work in an iterative manner to evaluate launch and orbital operations

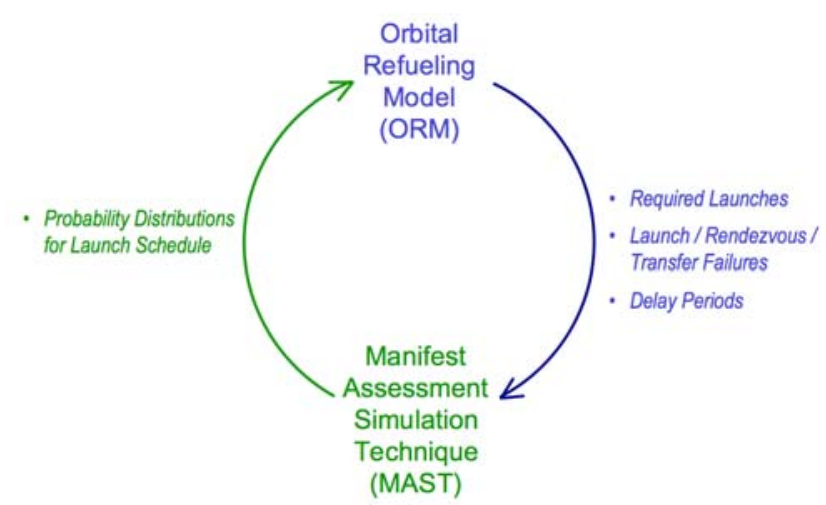

Figure 2. Iterative Simulation 
for a variety of launch concepts. Figure 2 details the basic operation of the simulation.

The Orbital Refueling Model (ORM) is a Monte Carlo model that evaluates the performance and reliability of the overall operations. The ORM has a flexible interface that allows missions and operations to be interactively structured. Using this interface, options for the number of launches, vehicles types, and scope of operations are defined. Probability distributions for operational reliability of each mission phase are also defined within the model. The ORM then specifically simulates each event in the overall concept of operations, including the outcome based on the input reliability distributions, and possible delays.

The ORM structure for the concept described in this paper is shown in Figure 3. In the proposed concept of operations, the first launch is of the EDS. If this launch is a success, then the EDS is placed in orbit and the refueling process begins. If the launch is a failure, then the overall mission fails. Following the EDS launch, a series of tanker flights are launched. The number of planned tanker flights is an input to the model. Each tanker launches, rendezvous with the EDS and transfers propellant in series. Each flight can fail on launch, rendezvous, or transfer. If a tanker flight fails, additional replacement flights can be attempted, if that option is selected in the model. If all tanker flights are successfully completed, then the model moves to the next stage. If the maximum number of tanker flight attempts is reached before the required number of tanker flights has been successfully achieved, then the overall mission fails. If all tanker missions are successfully completed, the Lunar Lander and Crew Module are then launched in series. Each of these flights is critical; if they fail in any part of the operations the overall mission fails.

In addition to the technical success for each event, the ORM also simulates and tracks the schedule for each successive event. The EDS has a limited lifetime, which starts when the element successfully reaches orbit. If, in a given run, all other required launches are not completed within the defined EDS lifetime, the overall mission is considered a failure.

Results of the ORM simulation are integrated across Monte Carlo runs to produce a distribution for the overall probability of success for the mission concept. The metric represents the overall probability that a fully fueled EDS, Lander, and Crew Module will be assembled in LEO and then proceed to Trans Lunar Injection (TLI).

Probability distributions for the schedule of each launch event evaluated in ORM are developed using the other component of the simulation - MAST. MAST is a discrete event simulation (DES) model, built using Arena by Rockwell Automation, which evaluates the launch and processing operations for each launch in a sequence and the

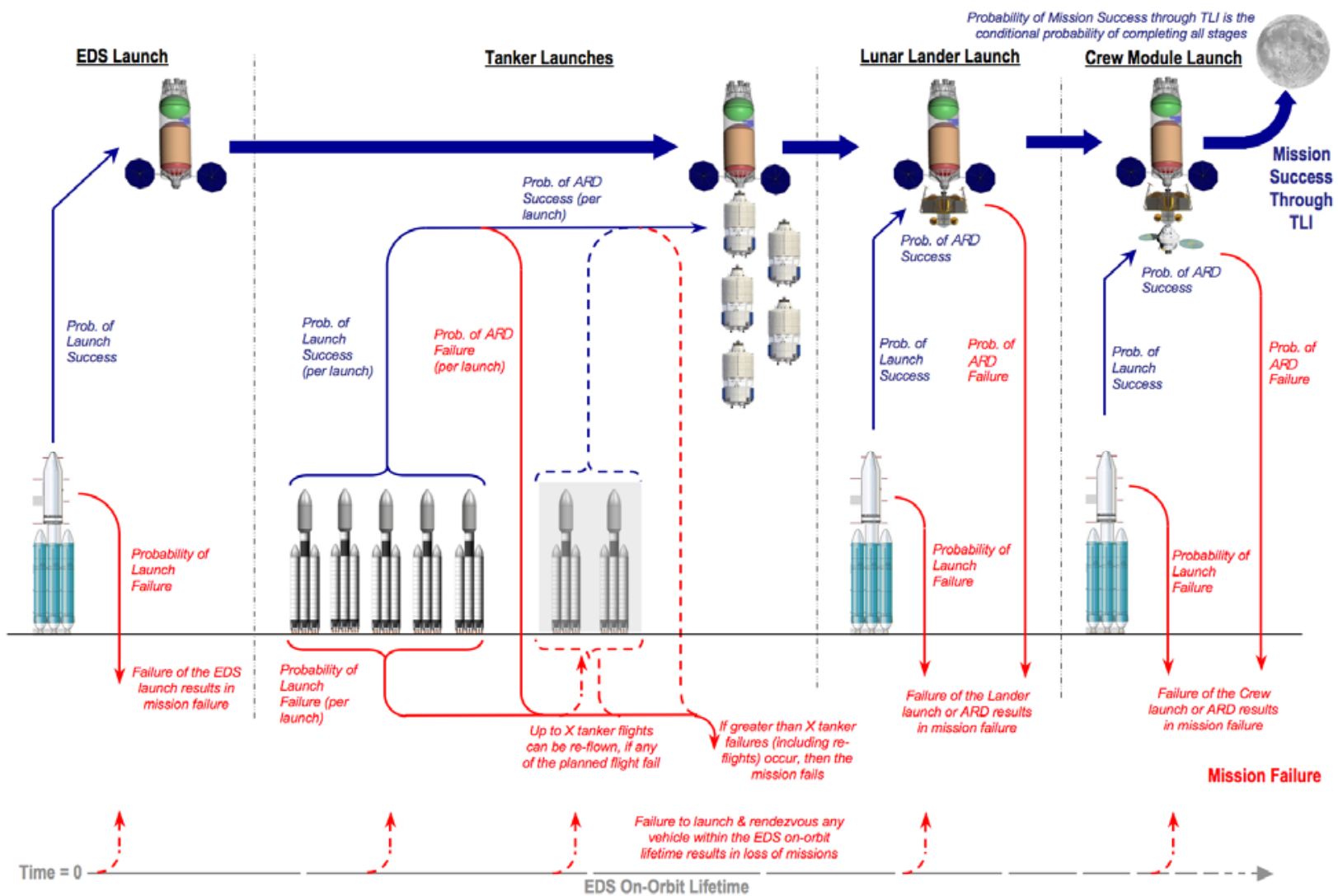

Figure 3. Simulation Flow of On-Orbit Refueling

4

American Institute of Aeronautics and Astronautics 
interactions between launches. ORM feeds MAST data for each planned launch in a given run, including a nominal schedule and delays imposed by failures. MAST takes that data and, based upon the results of its launch countdown simulation, feeds back a probability distribution for the achieved launch date for each launch.

The scope of MAST includes all of the processes shown in Figure 4. For this assessment we assumed that all launch vehicles are in a state of readiness (ready to begin launch countdown) when we commit to launch of the EDS. The practicality of this assumption was not addressed by our analysis. Launch countdowns are modeled using a cyclical event construct that matches the nature of launch vehicle countdowns ${ }^{6}$. There are discrete points in time in which decisions are made to continue on with the launch countdown or declare a launch delay of 1 or more days. These include a

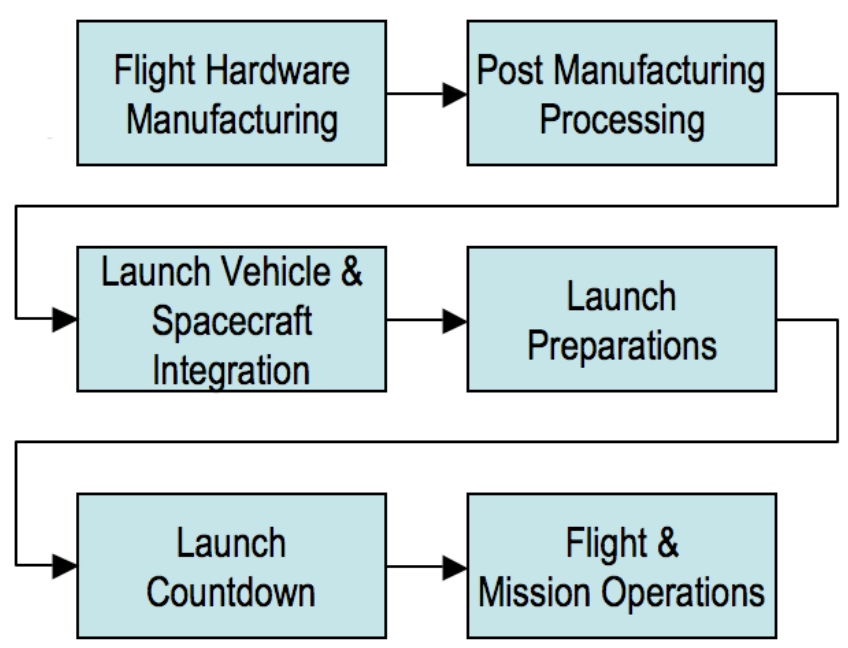

Figure 4. Scope of MAST Simulation launch minus 2 days review, a launch minus 1 day review, a Mission Management Team (MMT) decision to proceed with propellant fueling, the propellant fueling operations, the post fueling operations including astronaut ingress into the space vehicle if appropriate, an MMT decision to launch, a terminal countdown controlled by a ground launch sequencer, and an engine start sequence.

Depending upon when a delay is declared will, at least in part, determine where one will recycle back to in the countdown for the next attempt. For example, delays that occur after fueling has been initiated will have to recycle at least back to the MMT fueling decision point. The magnitude of the delay is dependent upon the initiating cause of the delay. For example, weather delays tend to be one day, whereas flight hardware delays tend to be more than one day.

Delay probabilities and durations are based on an analysis of historical analogs. Complete history files of launch countdowns for various vehicle types, including information on every launch delay (i.e., when the delay occurred, its reason, its duration, and where the countdown was re-entered for the next attempt) were evaluated in order to produce this data. If existing vehicles are used in the simulations, these datasets are directly used in the simulation.

If new proposed launch vehicles are used in the simulation, flight hardware launch delay probabilities and duration estimates are developed by decomposing the vehicles into their major components. This allows the ability to take into consideration the quantity and complexity of the flight hardware relative to the space shuttle and other vehicles in the database ${ }^{7,8}$.

\section{Cases}

Using the simulation models described above, the overall reliability of the EDS re-fueling concept was evaluated. Several variables and their impact on overall mission reliability were evaluated as part of this effort. Those variables were:

1. Number of Propellant Flights - The primary variable in this analysis is the number of tanker flights that are required to deliver propellant to the EDS. As part of this analysis, the number of flights was varied from zero to ten. The number of flights actually required will vary based on the desired cargo capacity landed on the moon and the achieved boil-off rate for propellant. For a capacity similar to that provided by the proposed Constellation architecture, it is anticipated that eight flights will be required. While at least one planned tanker flight will always be required to support this specific scenario, the zero tanker case was included in this analysis, because the results for that case will be representative of the reliability of a system in which a permanent on-orbit fueling depot is used (because in that case as many tanker flights as are required to deliver propellant could be launched prior to the launch of the EDS).

2. Launch Reliability of the Propellant Launch System - The second variable is the launch reliability of the launch system used to supply propellant to the EDS. For this analysis, launch reliabilities of $0.90,0.95$, and 0.985 were investigated. These reliabilities span the estimated range of expected reliability from new launch systems through matured launch systems. 
3. EDS Lifetime - The third major variable is the EDS LEO lifetime. This is the total time that the EDS can spend on orbit prior to TLI before the system fails. For this analysis on-orbit lifetimes of 30, 60, 90, and 120 days were investigated. 120 days is considered to be the practical limit from a technical standpoint. In addition, beyond that point, the MMOD risk outweighs any gains in lifetime risk.

4. Number of Available Spare Tanker Flights - The fourth variable that was analyzed was the number of spare tanker flights that are available and ready to fly. For this analysis, values of 0,1 , and 2 were evaluated. Two spare tanker launches covers almost all reasonable loss scenarios.

5. Reliability of the Automated Rendezvous, Docking, and Propellant Transfer - A range of reliabilities was evaluated for the on-orbit activities of each propellant refueling flight. A range of ARD and Transfer reliabilities from 0.95 to 0.99 were evaluated.

6. Availability of Ground Processing and Launch Infrastructure for Refueling Flights - The results of the MAST simulation are heavily influenced by the assumed availability of ground processing and launch infrastructure, particularly the number of available launch pads. For this analysis two independent cases were evaluated for propellant vehicle launch pad availability. The first case assumed that all propellant vehicles would be launched from Cape Canaveral and that there would be three launch pads available for the propellant flights. This was referred to as the 'constrained' case. The other case, referred to as the 'unconstrained' case, assumed that propellant vehicles could be launched from any site world-wide and that there was no limit on the number of available launch pads.

For the cases detailed in this paper, certain other parameters were held constant. The most significant is the launch reliability for each of the three dedicated hardware flights (i.e., EDS, Lunar Lander, and Crew Module), which was assumed to be 0.985 .

\section{Results}

The overall probability that a mission would reach TLI is the main output metric of interest for the simulation. Failure to reach TLI included all potential failure modes, including hardware failures and EDS on-orbit lifetime failures. This metric also accounted for the possibility of re-flying failed tanker flights.

Initial analysis of results showed that four of the six variables described above had a primary impact on the overall system reliability. Those variables were: the Number of Propellant Flights, the Launch Reliability of the Propellant Launch System, the EDS Lifetime, and the Number of Available Spare Tanker Flights. The impact of these variables on reliability is discussed below. The impact of the other two variables, the Reliability of the Automated Rendezvous, Docking and Fueling and the Availability of Launch Infrastructure, were found to have only a relatively minor impact on the overall reliability. The results discussed in this paper are limited to changes in the primary variables. Results for all cases shown below assume that the ARD \& Refueling reliability is 0.985 and that the ground and launch infrastructure is the 'Constrained' case.

The basic results for this metric across all runs are shown in Figures 5-7. Figure 5 show results for cases with zero available spare tanker flights. Figure 6 shows results for cases with a single spare tanker flight available. Figure 7 shows results for cases with two spare tanker flights available. In each figure a number of lines are shown to indicate the system reliability across the range of potential number of tanker flights. Individual lines are shown for different combinations of EDS lifetime and propellant launch system reliability.

The results of the simulation indicate that, in order to achieve overall mission reliabilities that are even close to the Heavy-lift Launch Vehicle (HLV) solutions, a refueling operation will have to employ an EDS with a long onorbit lifetime and will likely have to allow for replacement tanker flights. Each of the variables evaluated in this analysis are significant contributors to overall system reliability through TLI. For comparative purposes the overall reliability to TLI of the Constellation architecture is projected to be approximately 0.89 .

The number of tanker flights that are required is one of the primary drivers of overall system reliability, particularly at lower value of launch reliability. However, for a given class of launch vehicle the number of tankers will be dictated by the required amount of propellant. It is anticipated that employing a 25metric ton class launch vehicle, that between seven and nine tanker flights would be required to enable a lunar mission with a capacity similar to that provided by the Constellation architecture. 

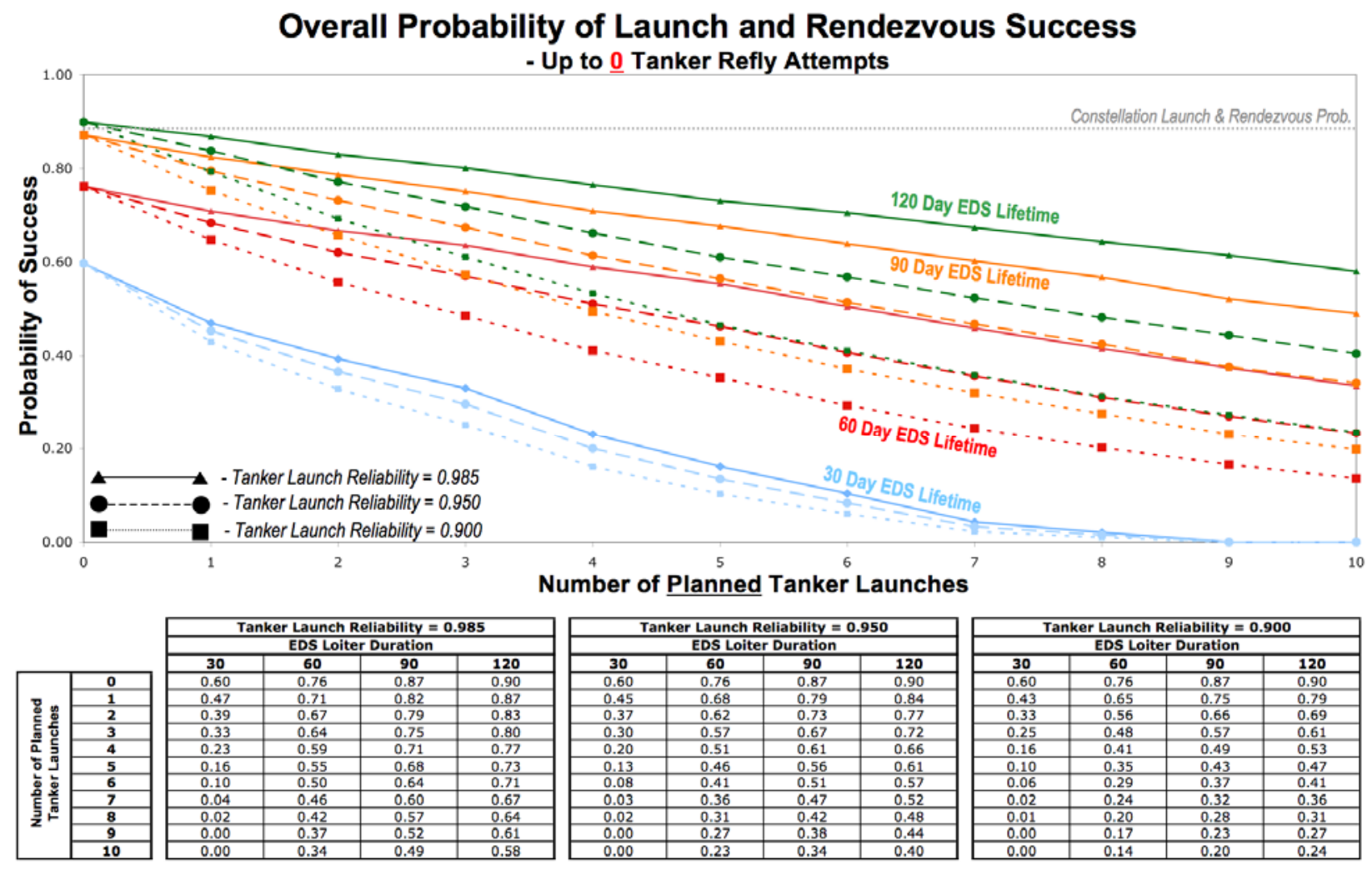

Figure 5. Simulation Results for 0 Available Tanker Refly Attempts
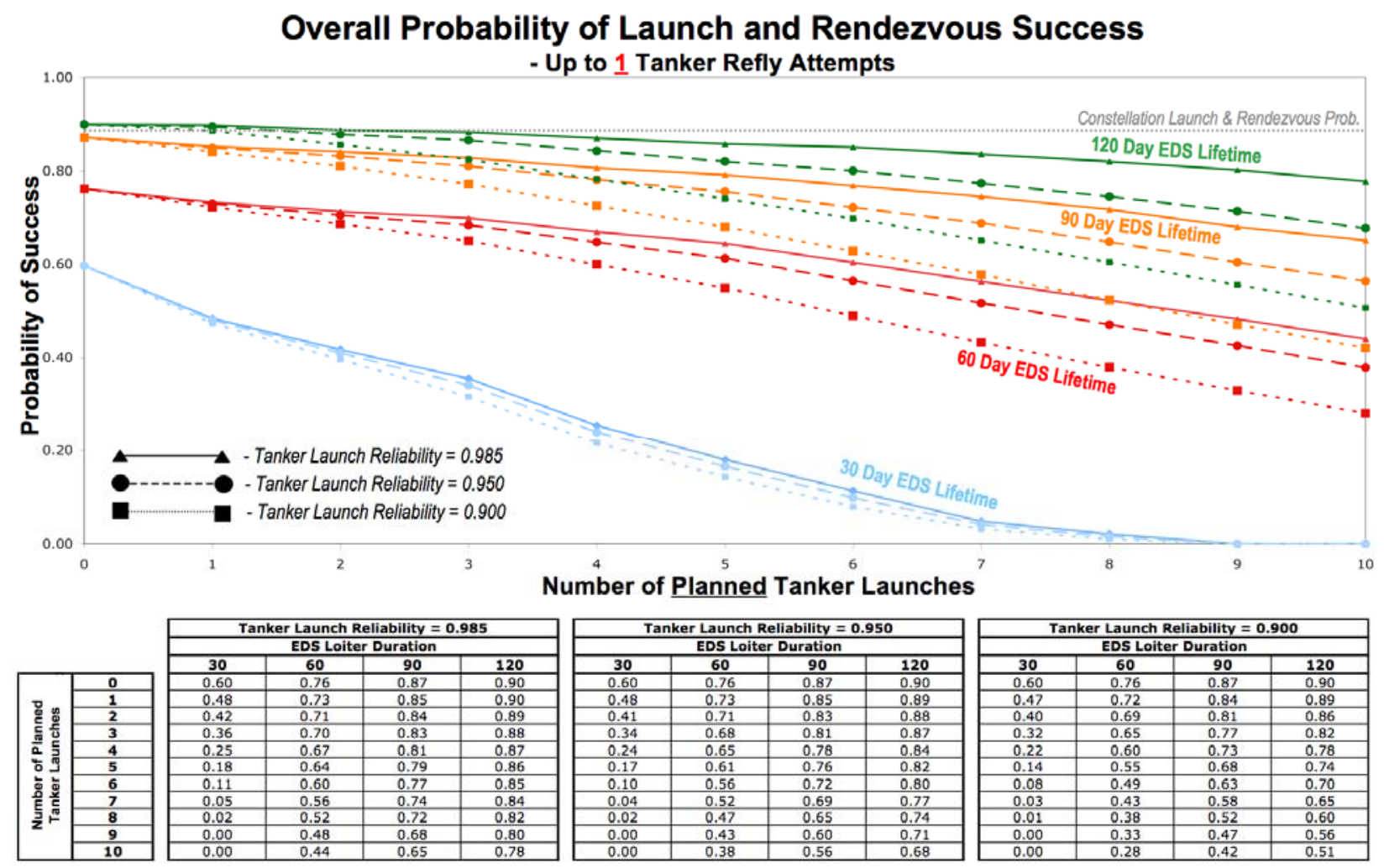

Figure 6. Simulation Results for 1 Available Tanker Refly Attempt

7

American Institute of Aeronautics and Astronautics 


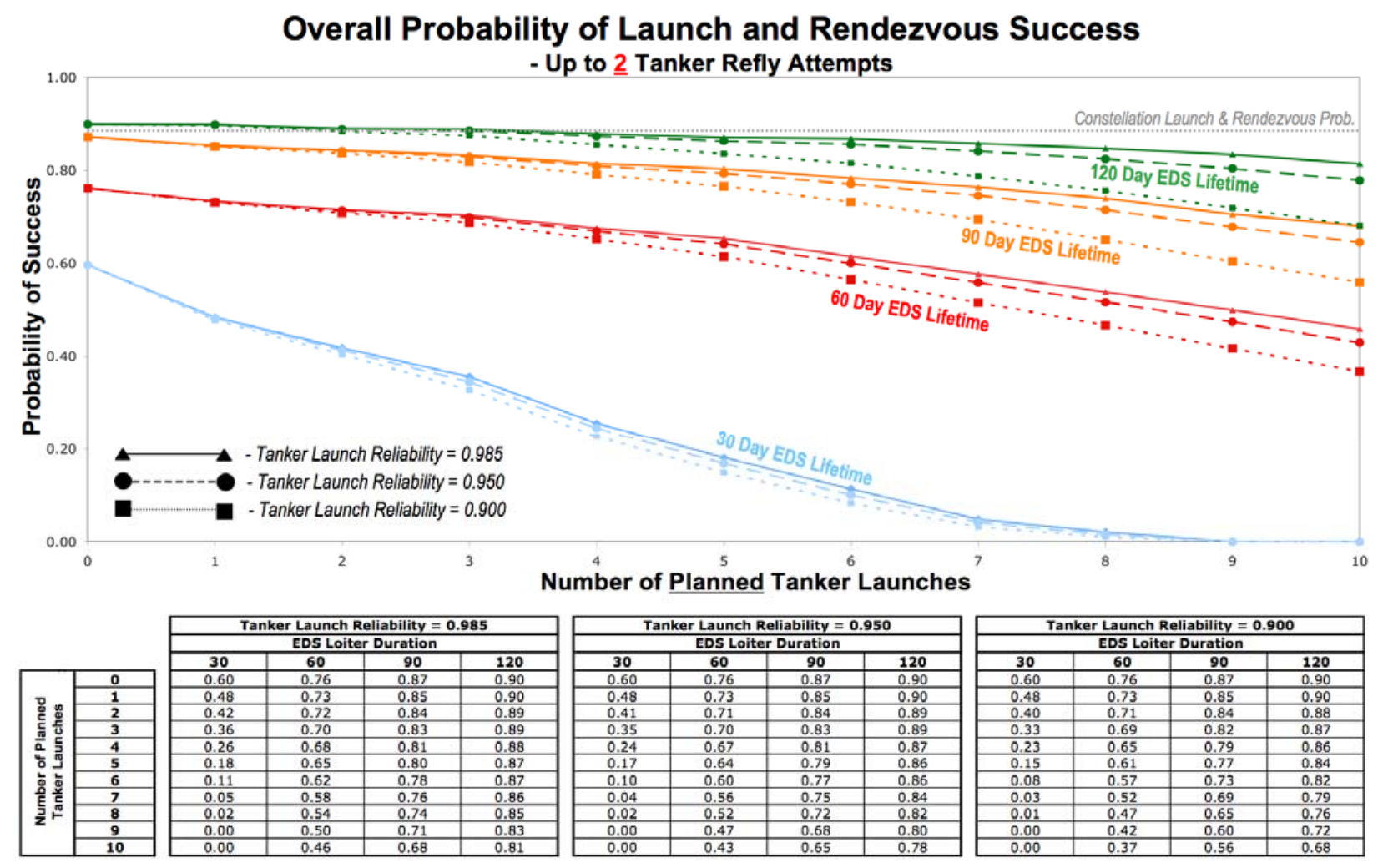

Figure 7. Simulation Results for 2 Available Tanker Refly Attempts

The second major contributor to overall system reliability is the availability of launch-on-need spare tanker flights to replace tanker flights that fail launch, rendezvous, docking, or propellant transfer. For the case referenced above with eight tanker flights and a tanker launch reliability of 0.985, at a 120-day EDS lifetime, the availability of a single spare tanker increases the overall reliability from 0.64 to 0.82 . A second spare tanker flight further increases the overall reliability to 0.85 . At lower levels of tanker vehicle launch reliability the impact of spare tanker flights is even more pronounced. For the same case with tanker launch reliability of 0.90 , the overall system reliability with no spare tankers is 0.31 . A single spare flight increases the reliability to 0.60 and a second spare to 0.76 .

The reliability of the tanker launch system is a major contributor to overall reliability, if no spare tanker flights are available. In this case the reliability of the tanker is critical because a failure in any single flight leads to overall system failure. When spare tankers are available, however, the impact of tanker launch reliability is reduced, since any given flight can be replaced in the event of a failure.

In order to approach the reliability projected for the Constellation system, both a long EDS on-orbit lifetime and spare tanker flights will have to be employed. With eight tanker launches and a tanker launch reliability of 0.985, if there is a 120-day EDS lifetime and one spare tanker flight, the projected reliability through TLI is 0.82 . This is comparable to the projected Constellation reliability of 0.89 .

\section{Forward Work \& Conclusion}

There are several extensions to the current model that are being developed to further support the analysis of propellant refueling concepts. One area of interest is the analysis of the reliability of permanent, on-orbit propellant refueling depots. This concept has the ability to reduce some of the risks described in this paper but could have significantly larger costs. Another area of development involves the extension of the MAST model to include manufacturing constraints. The infrastructure for EELV manufacturing is limited and delays in the availability of launch vehicles could have a major impact of refueling concepts. Finally, significant work is currently being conducted to extend the reliability analysis of on-orbit refueling concepts to include MMOD risk and the risk of onorbit system failure. Evaluation of these risks is critical since one of the primary findings in the current analysis is that long on-orbit system lifetimes will be required to support refueling concepts. 
Since the analysis described in this paper was completed in support of the Augustine Commission ${ }^{1}$, the tools that are described have been used to further support analysis of potential architectures. In particular, the tools have been used as part of the 'Human Exploration Framework Team (HEFT)' effort to evaluate possible implementations of the "Flex-Path" architecture and to inform future national space policy. Propellant refueling scenarios are a major focus of current analysis and the evaluation of the reliability of those scenarios will be important in establishing a future direction for human space flight.

In order to achieve reliabilities that approach those for the exclusively HLV-based architecture, the EDS on-orbit lifetime will have to be as long as possible. In the results of this analysis, any periods of less than 120 days resulted in poor overall reliability. In addition, in order to approach HLV-based reliability, there will likely have to be some possibility of re-flying lost tanker flights. Allowing a single replacement flight significantly increased the overall reliability. Adding a second flight did not have as dramatic an effect. However, a second flight was required to achieve reliabilities that were close to the HLV architecture. It is possible that the overall mission reliability could be increased without replacement flights, if the tanker launch reliability could be significantly increased. However, a significant improvement in reliability is likely to require a more advanced launch vehicle.

The results presented in this paper represent the performance of a refueling concept using an EDS as the propellant depot for a lunar mission. Other concepts, such as a permanent on-orbit propellant depot may have significantly different mission reliabilities. In addition, the use of propellant refueling for missions to other destinations may result in very different reliabilities. The simulation models described in this paper have the capability of evaluating and comparing the reliability of these alternate scenarios.

\section{References}

\footnotetext{
${ }^{1}$ Review of Human Spaceflight Plans Committee, Seeking a Human Spaceflight Program Worthy of a Great Nation, National Aeronautics and Space Administration, 2009, Web. 7 July 2010. <http://www.nasa.gov/offices/hsf/home/index.html>.

${ }^{2}$ Foust, J., “Propellant Depots: An Idea Whose Time Has (Almost Come),” The Space Review, May 12, 2008.

3 Constellation, National Aeronautics and Space Administration, 2010. Web. 9 July 2010. <http://www.nasa.gov/mission_pages/constellation/main/>

${ }^{4}$ Young, J.J., Thompson, R.W., and Wilhite, A.W., "Architecture Options for Propellant Resupply of Lunar Exploration Vehicles,” Proceedings of AIAA SPACE 2006 Conference, 2006.

${ }^{5}$ Goff, J.A., Kutter, B.F., Zeglar, F., Bienhiff, D., Chandler, F., and Marchetta, J., "Realistic Near-Term Propellant Depots: Implementation of a Critical Spacefaring Capability”, AIAA SPACE 2009, 2009.

${ }^{6}$ Cates, G.R. and Mollaghasemi, M., "Supporting the vision for space with discrete event simulation," Proceedings of the IEEE 2005 Winter Simulation Conference, 8913370, 2005.

${ }^{7}$ Cates, G.R., Stromgren, C., and Cirillo, W.M., "Low earth orbit rendezvous strategy for lunar missions," Proceedings of the IEEE 2006 Winter Simulation Conference, 1-4244-0500-9, 2006.

${ }^{8}$ Stromgren, C., Cates, G.R., and Cirillo, W.M., "Launch Order, Launch Separation, and Loiter in the Constellation 11/2Launch Solution,” 2009 IEEE Aerospace Conference, 978-1-4244-2621-8, 2009.
} 